\title{
Classificação das Patentes em Universidades Federais na Escala TRL (Technology Readiness Level): estudo de caso a partir da Norma ISO 16290:2013
}

\author{
Classification of Patents in Federal Universities in the TRL Scale (Technology \\ Readiness Level): case study from the ISO 16290:2013 Standard
}

\author{
Mariana Eleutério Ribeiro ${ }^{1}$ \\ Irineu Afonso Frey ${ }^{1}$ \\ Paola Azevedo ${ }^{1}$ \\ ${ }^{1}$ Universidade Federal de Santa Catarina, Florianópolis, SC, Brasil
}

\begin{abstract}
Resumo
A transferência das patentes registradas pelas universidades, à sociedade, é fundamental para que possam, efetivamente, se tornarem inovação. Uma linguagem comum que facilite a extração de informações dos pesquisadores sobre suas descobertas comercializáveis requer precisa percepção do grau de maturação da tecnologia, em que se destaca a escala TRL (Technology Readiness Level). Frente a esse contexto, este artigo tem como objetivo enquadrar os pedidos de depósito de patentes de uma Universidade Federal na escala TRL. Por meio de uma pesquisa qualitativa de caráter exploratório e de estudo de caso, foi elaborado um protocolo de classificação baseado na Norma ISO 16290:2013 que gerou uma solução tecnológica (software) para cálculo de nivelamento na escala. O protocolo de classificação foi respondido pelos respectivos inventores de janeiro a setembro de 2020. O software elaborado contribuiu para a classificação de patentes em universidades federais, com base em parâmetros científicos, e elucidou as potencialidades de transferência tecnológica de uma universidade federal, visto que os resultados revelaram uma predominância de patentes localizadas no nono nível da escala, com inconsistências a partir do quinto nível.
\end{abstract}

Palavras-chave: Propriedade Intelectual. TRL (Technology Readiness Level). Transferência de Tecnologia.

\begin{abstract}
The transfer of patents registered by universities to society is essential for them to effectively become innovation. A common language that facilitates the extraction of information from researchers about their marketable discoveries requires a precise perception of the degree of technology maturation, where the TRL (Technology Readiness Level) scale stands out. In view of this context, this article aims to classify the applications for filing patents from a Federal University on the TRL scale. Through a qualitative exploratory research and case study, a classification protocol based on the ISO 16290:2013 standard and a technological solution (software) for the calculation of leveling on the scale were elaborated. The classification protocol was answered by the respective inventors from January to September 2020. The software developed contributed to the classification of patents in federal universities, based on scientific parameters, and elucidated the potential for technological transfer of a federal university, as the results revealed a predominance of patents located at the ninth level of the scale, with inconsistencies from the fifth level.
\end{abstract}

Keywords: Intellectual Property. TRL (Technology Readiness Level). Technology Transfer.

Área Tecnológica: Propriedade Intelectual. Inovação e Desenvolvimento. 


\section{Introdução}

As universidades têm desempenhado um importante papel para a consolidação da economia do conhecimento, que tem feito dos ativos intangíveis grandes fontes de desenvolvimento dos países. Especificamente no âmbito das patentes, a difusão e a transferência desses ativos à sociedade são fundamentais para que possam efetivamente tornar-se inovação.

Nos países no topo do ranking da produção de patentes, como os Estados Unidos, as instituições de ensino superior representam uma pequena parcela, em razão do domínio das empresas privadas nesse mercado. No Brasil, verifica-se o oposto, uma vez que universidades federais têm competido paralelamente com as indústrias em quantidade de patentes depositadas (PÓVOA, 2010).

O objetivo comum das políticas de patentes das universidades brasileiras tem sido alcançar o equilíbrio entre as necessidades dos inventores, da instituição, dos patrocinadores da pesquisa e do público em geral. O processo de transferência de tecnologia tem como foco os resultados da pesquisa científica e os aspectos de licenciamento e comercialização que, em geral, apresentam como opções estratégicas a exploração própria da patente, o uso da patente para impedir sua exploração por terceiros, a transferência dos direitos a terceiros mediante compensação financeira, a concessão de licença a terceiros e o uso da patente na constituição de uma nova empresa (BEM-AMI, 2000).

Para apresentar os conceitos fundamentais sobre a relação universidade-empresa e os processos de transferência de tecnologia para o mercado, optou-se por realizar um resgate teórico que se inicia com Schumpeter, avançando na contribuição dos neoschumpeterianos, na caracterização dos sistemas de inovação e a teoria da Tríplice Hélice e sua atualização.

Plonski (1992) definiu as interações universidade-empresa como um modelo de arranjo interinstitucional entre organizações distintas (que possivelmente tenham finalidades diferentes) e que podem ocorrer de forma tênue, como na oferta de estágios profissionalizantes, ou forte, com pesquisa colaborativa, divisão de titularidades e, consequentemente, a repartição dos lucros provenientes da comercialização dos resultados de pesquisa. Já a transferência de tecnologia pode ser entendida como o processo pelo qual um conjunto de conhecimentos, habilidades e procedimentos aplicáveis aos problemas de produção são transferidos, por transação de caráter econômico, de uma organização e outra, ampliando a capacidade de inovação da organização receptora.

O papel do conhecimento na geração de inovações que impactam no desenvolvimento econômico é reconhecido desde Schumpeter com a publicação da sua Teoria do Desenvolvimento Econômico, na qual o autor propõe o entendimento do desenvolvimento econômico a partir das inovações, discutindo o modelo de fluxo circular da riqueza. Em sua abordagem, a inovação é vista como fator de desequilíbrio do qual decorrem as variações cíclicas da economia (LAPLANE, 2004).

Em sequência, autores neoschumpeterianos avançaram na compreensão do desenvolvimento econômico sustentado pela inovação como um processo evolucionário por dois caminhos teóricos. A primeira vertente, da Universidade de Yale (Estados Unidos), tem como principais autores os evolucionistas Nelson e Winter, que contribuíram pela incorporação dos conceitos evolucionários na Teoria Econômica. Por outro lado, a vertente da Universidade de Sussex 
(Reino Unido) trouxe a discussão de seus principais nomes C. Freeman, C. Perez, K. Pavitt, L. Sorte e G. Dosi sobre os processos de geração e propagação de novas tecnologias (POSSAS, 1989; AZEVEDO, 2016).

Sábato e Botana (1968) introduziram na América Latina uma estratégia de inserção da C\&T para o processo de desenvolvimento dos países. A partir do Triângulo de Sábato, novos modelos surgiram, como a Tríplice Hélice, que preconiza as relações entre universidade, empresas e governo, em que a universidade se ocupa do ensino e da pesquisa em um novo foco para o desenvolvimento de novas tecnologias, estimulando ambientes de inovação e a cultura empreendedora. Nesse processo, a transferência da tecnologia produzida na universidade para as empresas que poderão explorá-la economicamente está vinculada ao desenvolvimento de patentes e de seu licenciamento (ETZKOWITZ; LEYDESDORFF, 1997).

A literatura recente apresenta uma atualização do modelo da Tríplice Hélice, reconhecendo a existência de uma quarta e quinta hélices que movem os processos de inovação de um país. Conhecido como Quadruple Helix, o modelo de Carayannis e Campbell (2009) apresenta a quarta hélice como sendo o público baseado em mídia e em cultura, ou seja, uma evolução conjunta com a economia do conhecimento. Já o modelo que identifica a quinta hélice, conhecido como Quintuple Helix, reverbera na sustentabilidade, gerando um impacto positivo na sociedade como um todo (CARAYANNIS; BARTH; CAMPBELL, 2010). Este é baseado nos modelos das hélices anteriores e engloba os ambientes naturais da sociedade, como as comunidades locais, os fornecedores, os funcionários, os parceiros, os clientes, os acionistas, as autoridades públicas e as Organizações não Governamentais (ONGs) (LOZANO et al., 2019).

Thursby, Fuller e Thursby (2009) identificaram barreiras específicas no processo transferência de tecnologia para exploração econômica das patentes produzidas em universidades $e$ destacaram entre elas a falta de uma linguagem comum que facilite a extração de informações dos pesquisadores acerca de suas descobertas comercializáveis, o que muitas vezes não ocorre pela falta de percepção desse potencial. Outros estudos sobre realidades internacionais também destacam o papel fundamental dos pesquisadores na comunicação do invento para fins de comercialização (AGRAWAL, 2006; THURSBY; FULLER; THURSBY, 2009).

Nessa lacuna reside tanto a necessidade quanto a oportunidade de as universidades comunicarem às empresas suas tecnologias com potencial de comercialização. Essa avaliação requer a percepção do grau de maturidade da tecnologia, avaliação esta que se manifesta por meio de diferentes métodos de análise, entre eles, a escala TRL (Technology Readiness Level), uma escala de nove níveis em que o primeiro caracteriza a investigação básica ou ideia em desenvolvimento e o nono apresenta o sistema real finalizado e qualificado por meio de operações com êxito em missões (MANKINS, 1995).

A escala TRL foi publicada pela primeira vez em 1989 por Sadin (1989), após o desenvolvimento e a implementação pela NASA na década de 1970. Verifica-se seu amplo uso como metodologia para qualificação de tecnologias mundialmente, em especial nas indústrias aeroespacial, petróleo e gás e de segurança. Desde 2001, a TRL é utilizada em todos os novos programas de compras e aquisição tecnológica do Departamento de Defesa dos Estados Unidos (OLECHOWSKI; EPPINGER; JOGLEKAR, 2015). Além disso, a escala tem sido considerada como critério de exigibilidade para concessões de financiamento no Programa de Pesquisa e Inovação Horizonte 2020 da Comissão Europeia (EUROPEAN COMMISSION, 2015). 
Em 2013, essa metodologia de medição de prontidão tecnológica passou por uma importante fase, que foi a padronização pela International Organization for Standardization (ISO). A Norma ISO 16290, publicada em 2013, padroniza a aplicação da TRL e apresenta explicações detalhadas a respeito dos diferentes termos e conceitos utilizados ao longo da sistematização dos critérios de classificação para cada um dos nove níveis da escala.

Na Universidade Federal de Santa Catarina (UFSC), desde o ano de 2017, há o campo "TRL" a ser preenchido pelos pesquisadores no Sistema Integrado de Gerenciamento de Projetos de Pesquisa e de Extensão, utilizado para tramitação dos projetos de pesquisa. Entretanto, os pesquisadores não contam com auxílio para calcular exatamente em qual nível de maturação encontra-se a propriedade intelectual de seus projetos. Também não há ferramenta que classifique o nível da maturação tecnológica da propriedade intelectual da universidade.

Assim, considerando o exposto acerca do tema e a necessidade da universidade, este artigo tem o objetivo de classificar na escala TRL os pedidos de registro de patente da Universidade Federal de Santa Catarina. Para isso, foi elaborado um protocolo de classificação com base na Norma ISO 16290:2013 e, a partir do qual, foi desenvolvido o software TRLCalc, ferramenta para cálculo do nível de prontidão de uma tecnologia na escala TRL.

\section{Metodologia}

Esta pesquisa caracteriza-se como uma pesquisa qualitativa, exploratória e estudo de caso, que objetivou-se enquadrar os pedidos de depósito de patentes de uma Universidade Federal na escala TRL, por meio da elaboração de um protocolo de classificação baseado na Norma ISO 16290:2013 gerando uma solução tecnológica (software) para cálculo de nivelamento na escala

Elaborou-se um protocolo de classificação baseado na Norma 16290:2013, que apresenta a caracterização esperada para cada um dos nove níveis da escala. Para isso, extraiu-se desse documento afirmações absolutas que caracterizam cada nível da escala TRL, que foram reescritas para que seu conteúdo se formatasse como uma pergunta que pudesse ser respondida com "Sim" ou "Não" pelos inventores das patentes registradas na UFSC. Após serem sintetizadas para eliminação de ambiguidades e duplicidades, chegou a uma lista de 24 perguntas que compõem o protocolo de classificação utilizado neste estudo:

1) Os princípios básicos de sua pesquisa já foram observados e relatados por meio de pesquisas acadêmicas?

2) As potenciais aplicações da tecnologia ${ }^{1}$ já foram identificadas?

3) Os requisitos de desempenho ${ }^{2}$ já foram especificados?

4) O conceito da tecnologia e/ou da aplicação já foi formulado?

5) As aplicações práticas já podem ser identificadas?

6) Os requisitos de desempenho do elemento 3 já foram identificados de forma consistente?

\footnotetext{
${ }^{1}$ Aplicação de conhecimentos científicos, ferramentas, técnicas, artesanato, sistemas ou métodos de organização, a fim de resolver um problema ou alcançar um objetivo.

${ }^{2}$ Conjunto de parâmetros que devem ser satisfeitos pelo elemento.

${ }^{3}$ Item ou objeto sob consideração para a avaliação da prontidão tecnológica
} 
7) A função crítica ${ }^{4}$ já foi caracterizada?

8) Já foi realizada a prova ${ }^{5}$ da função crítica (por análise, modelagem, simulação ou experimentação)?

9) Os requisitos de desempenho do elemento são consistentes em relação a qualquer conceito ou aplicação formulada?

10) Os objetivos estão definidos em relação ao estado atual da arte?

11) Já foi realizada a verificação funcional e/ou breadboard ${ }^{6}$ em ambiente de laboratório ${ }^{7}$ ?

12) O ambiente relevante 8 já foi definido?

13) A demonstração das funções críticas do elemento já foi iniciada no ambiente relevante?

14) O desempenho do teste em ambiente relevante está de acordo com as previsões?

15) Os testes de breadboard foram executados com sucesso?

16) Os requisitos de desempenho do elemento podem ser considerados consolidados, levando em conta os testes de verificação?

17) $\mathrm{O}$ desenvolvimento do elemento pode ser totalmente protegido por causa da incerteza resultante dos efeitos de dimensionamento?

18) As funções críticas do elemento foram demonstradas em ambiente relevante?

19) O desempenho geral do elemento foi demonstrado em ambiente relevante?

20) É possível estabelecer um cronograma de desenvolvimento para o elemento?

21) O modelo corresponde totalmente aos aspectos planejados quando operado em um ambiente operacional ${ }^{9}$ ?

22) O modelo está testado em ambiente operacional e pronto para o ambiente real?

23) Se o elemento qualificado for um componente, pode ser integrado no sistema final $e$ colocado em serviço para uma missão designada?

24) O elemento foi bem-sucedido em ambiente operacional real?

Esse questionário foi elaborado no Google Forms e enviado aos 282 inventores relacionados a 120 pedidos de depósito de patentes, os quais foram extraídos do relatório enviado pela SINOVA/UFSC em novembro de 2019. As respostas foram coletadas entre janeiro e setembro de 2020. O estudo contemplou, por acessibilidade, aproximadamente $40 \%$ dos pedidos de depósito de patente.

\footnotetext{
${ }^{4}$ Função obrigatória que requer verificação de tecnologia (ocorre quando o elemento ou os componentes do elemento são novos e não podem ser avaliados confiando em realizações anteriores ou quando o elemento é usado em um novo domínio, como novas condições ambientais ou um novo uso específico não previamente demonstrado)

${ }^{5}$ Teste que deve incluir estudos analíticos para definir a tecnologia em um contexto apropriado e experimentos ou medições baseadas em laboratório para apoiar fisicamente as previsões e modelos analíticos.

${ }^{6}$ Modelo físico projetado para testar a funcionalidade e adaptado à necessidade de demonstração.

${ }^{7}$ Ambiente controlado necessário para demonstrar os princípios subjacentes e o desempenho funcional. O ambiente de laboratório não necessariamente aborda o ambiente operacional, que por sua vez é o conjunto de condições naturais e induzidas que restringem o elemento a essas condições.

${ }^{8}$ Subconjunto mínimo do ambiente operacional que é necessário para demonstrar funções críticas do desempenho do elemento em seu ambiente operacional, que, por sua vez, é o conjunto de condições naturais e induzidas que restringem o elemento a essas condições.

${ }^{9}$ Conjunto de condições naturais e induzidas que restringem o elemento a essas condições.
} 


\section{Resultados e Discussão}

Nesta seção, apresenta-se a elaboração do software TRLCalc para nivelamento na TRL de uma determinada tecnologia, a partir das instruções do protocolo de classificação elaborado nesta pesquisa, de acordo com a Norma ISO 16290:2013 e a utilização do software para análise e classificação das patentes da Universidade Federal de Santa Catarina. O desenvolvimento do software TRLCalc considerou instruções básicas e complementares.

As instruções básicas consistem no atendimento progressivo dos requisitos apresentados pelo protocolo de classificação, que, na medida em que são sucessivamente atendidos, elevam o resultado entre os níveis da escala TRL. As instruções complementares atendem às possibilidades não incluídas nas instruções básicas e consistem em situações em que é necessário apresentar ao usuário do software informações complementares ao resultado apresentado. Essa necessidade nasce a partir de respostas negativas que fazem com que os requisitos de determinado nível da escala não estejam completamente preenchidos, porém, o resultado sofre a influência tendente aos níveis mais elevados quando essa situação é sucedida de respostas predominantemente positivas.

Assim, se as perguntas 1 a 3 obtêm pelo menos uma resposta positiva, a tecnologia estará enquadrada no primeiro nível da escala. Não é um resultado esperado que as três perguntas apresentem "Não" como resposta.

Se as perguntas 1 a 6 têm "Sim" como resposta e nenhuma resposta positiva entre o bloco de perguntas 7 a 10, o resultado permanecerá na TRL 2, porém, se pelo menos uma resposta for positiva, o resultado avançará para TRL 3.

A pergunta 11 direciona o resultado para que permaneça no terceiro nível ou eleve-se ao quarto. Se as condições para estar no terceiro nível estão atendidas e a resposta é positiva ao item 11, o resultado será o quarto nível da escala. Se for negativo, o resultado permanecerá no terceiro nível.

O próximo bloco de perguntas (12 a 17) direcionará o resultado para que este permaneça no quarto nível ou eleve-se ao quinto nível da escala. Se as condições para estar no quarto nível estiverem atendidas e a resposta for positiva em pelo menos uma das perguntas entre 12 e 17, o resultado será o quinto nível da escala. Se todas forem negativas, o resultado permanecerá no quarto nível.

As perguntas (18 a 20) direcionarão o resultado para que permaneça no quinto nível ou eleve-se ao sexto nível da escala. Se as condições para estar no quinto nível estiverem atendidas e a resposta for positiva em pelo menos uma das perguntas entre 18 e 20, o resultado será o sexto nível da escala. Se todas forem negativas, o resultado permanecerá no quinto nível.

A pergunta 21 direcionará o resultado para que permaneça no sexto nível ou eleve-se ao sétimo. Se as condições para estar no sexto nível estiverem atendidas e a resposta for positiva no item 21 , o resultado será o sétimo nível da escala. Se for negativo, o resultado permanecerá no sexto nível.

A pergunta 22 direcionará o resultado para que permaneça no sétimo nível ou eleve-se ao oitavo. Se as condições para estar no sétimo nível estiverem atendidas e a resposta for positiva no item 22 , o resultado será o oitavo nível da escala. Se for negativo, o resultado permanecerá no sétimo nível.

O alcance no nono nível da escala está condicionado aos resultados obtidos nas perguntas do bloco de 23 a 24, que direcionarão o resultado para que permaneça no oitavo nível ou 
eleve-se ao nono e último da escala TRL. Se as condições para estar no oitavo nível estiverem atendidas e a resposta for positiva em pelo menos uma das duas perguntas, o resultado será o nono nível da escala. Se forem ambas negativas, o resultado permanecerá no oitavo nível.

Primeiramente, verificou-se a possibilidade de as perguntas poderem ser respondidas predominantemente de forma positiva, principalmente as perguntas que sinalizam o nono nível da escala (perguntas 23 e 24) e de forma negativa a uma ou mais perguntas dos demais níveis. Ou seja, a hipótese de as respostas serem diferentes do esperado pela sequência da Norma ISO 16290:2013, que preconiza uma sequência de fatos que se sucedem e elevam-se na escala conforme completam etapas no desenvolvimento da tecnologia.

A questão 24 propõe ao usuário da calculadora avaliar se o elemento foi bem-sucedido em ambiente operacional real. Se essa resposta for positiva no caso prático, a tecnologia está indiscutivelmente em TRL 9, mesmo que o usuário responda negativamente alguma das demais questões, o que contraria o caráter sequencial da norma da ISO utilizada como referência. Assim, ao usuário que dessa forma proceder, a calculadora responderá TRL 9 e complementarmente qual nível está incompleto sob a perspectiva da ISO 16290:2013.

A mesma lógica foi aplicada aos sete demais agrupamentos de questões, até a TRL 8. Não se aplica, ao último agrupamento (questões 23 e 24), pois basta que uma delas esteja respondida positivamente para que o nono nível esteja atendido.

Após essa observação pelos agrupamentos de questões que caracterizam cada nível da escala, realizou-se a análise da combinação de respostas negativas entre esses agrupamentos, mantida a hipótese de que as questões que caracterizam o nível mais elevado da escala estarem respondidas positivamente. Por exemplo: na hipótese das questões que caracterizam a TRL 9 estarem respondidas positivamente, mas uma ou mais questões que caracterizam a TRL 1 e da TRL 2 estarem respondidas negativamente, o resultado na escala será TRL 9, com a mensagem complementar de que "Sob a perspectiva da Norma ISO 16290:2013, sua tecnologia cumpre requisitos que caracterizam a TRL 9, porém os requisitos da TRL 1 e TRL 2 estão incompletos". Essa lógica se manteve nos demais agrupamentos de questões.

Avançando para as demais possibilidades de respostas dadas às questões, pode ocorrer a seguinte combinação de acontecimentos: concomitantemente, os requisitos da TRL9 não serem atendidos e os da TRL 8 serem atendidos e algum outro não ser. Nesse caso, para estar no oitavo nível da escala, o modelo em análise deve estar testado em ambiente operacional e pronto para o ambiente real. No caso de algum outro nível da escala ter requisitos não satisfeitos, o usuário receberá como informação complementar ao resultado "TRL 8", explicando que, sob a perspectiva da Norma ISO 16290:2013, a tecnologia cumpre requisitos que caracterizam a TRL 8, porém os requisitos do nível cuja resposta negativa se refere, estão incompletos.

A partir dessas instruções básicas e complementares, o software TRLCalc foi construído na plataforma Eclipse e em linguagem Java com a proposta de apresentar-se como calculadora simples, limpa, confiável e de fácil utilização. Importante resgatar que as possibilidades dessa aplicação não são objeto deste trabalho.

Após acesso, o usuário encontra as questões dispostas na ordem de 1 a 24 , com as opções de resposta (Sim ou Não) em branco, para serem preenchidas, conforme mostra a Figura 1. Após o preenchimento de todas as respostas, o usuário encontra abaixo das questões dois botões: "Calcular TRL" e "Limpar". 
Existe alguma limitação da clareza das imagens para a proteção do software? Se sim, é importante esclarecer no corpo do texto.

Figura 1 - Tela principal do software TRLCalc

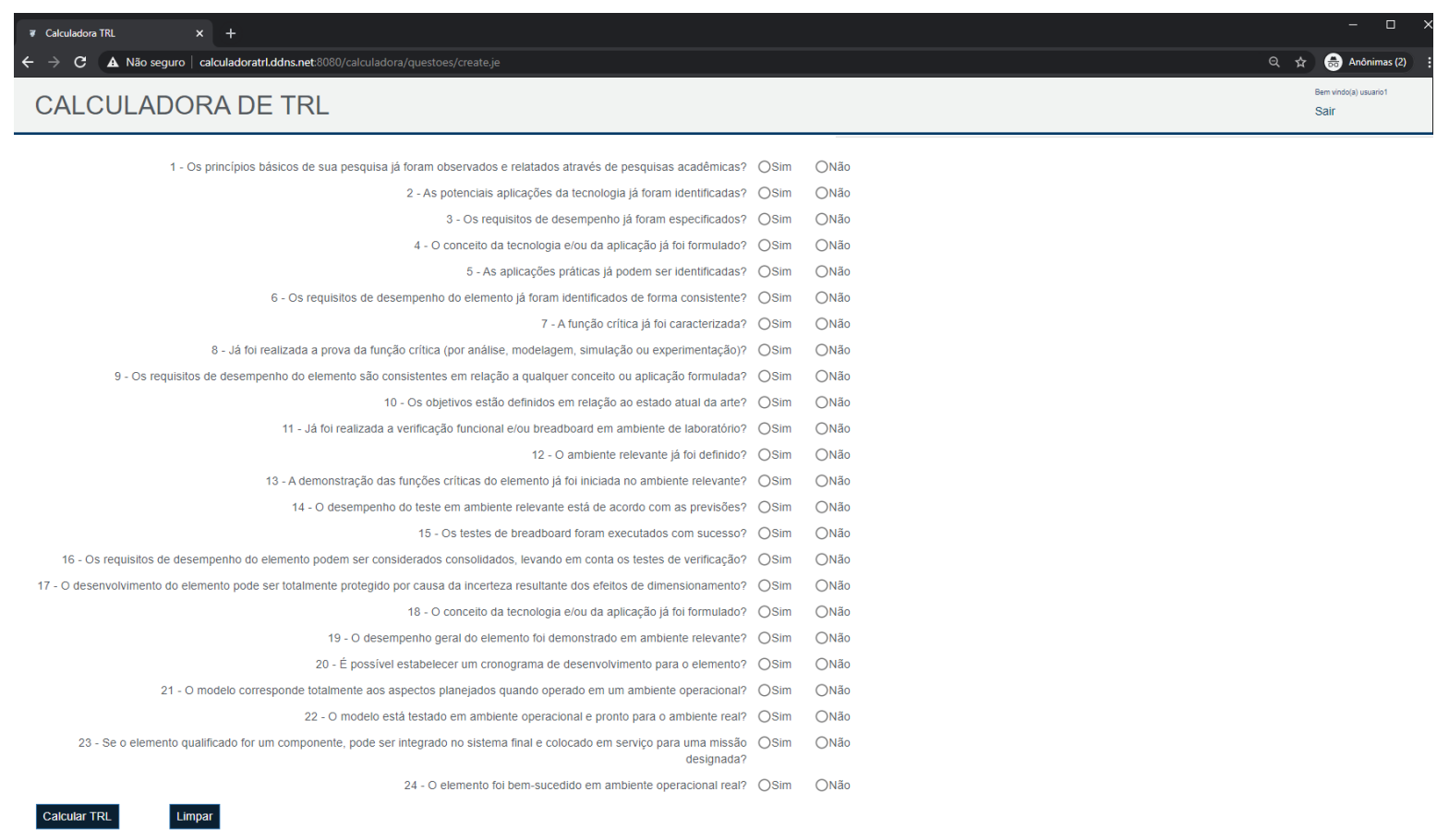

Fonte: Elaborada pelos autores deste artigo (2020)

Ao clicar no botão "Calcular TRL", o sistema gera uma resposta na escala TRL, conforme apresenta a Figura 2. Caso ocorra equívoco por parte do usuário no preenchimento dos dados, o sistema comunica a necessidade do ajuste, e só depois dessa alteração observa-se o resultado na escala.

Figura 2 - Simulação de resultado exato na escala

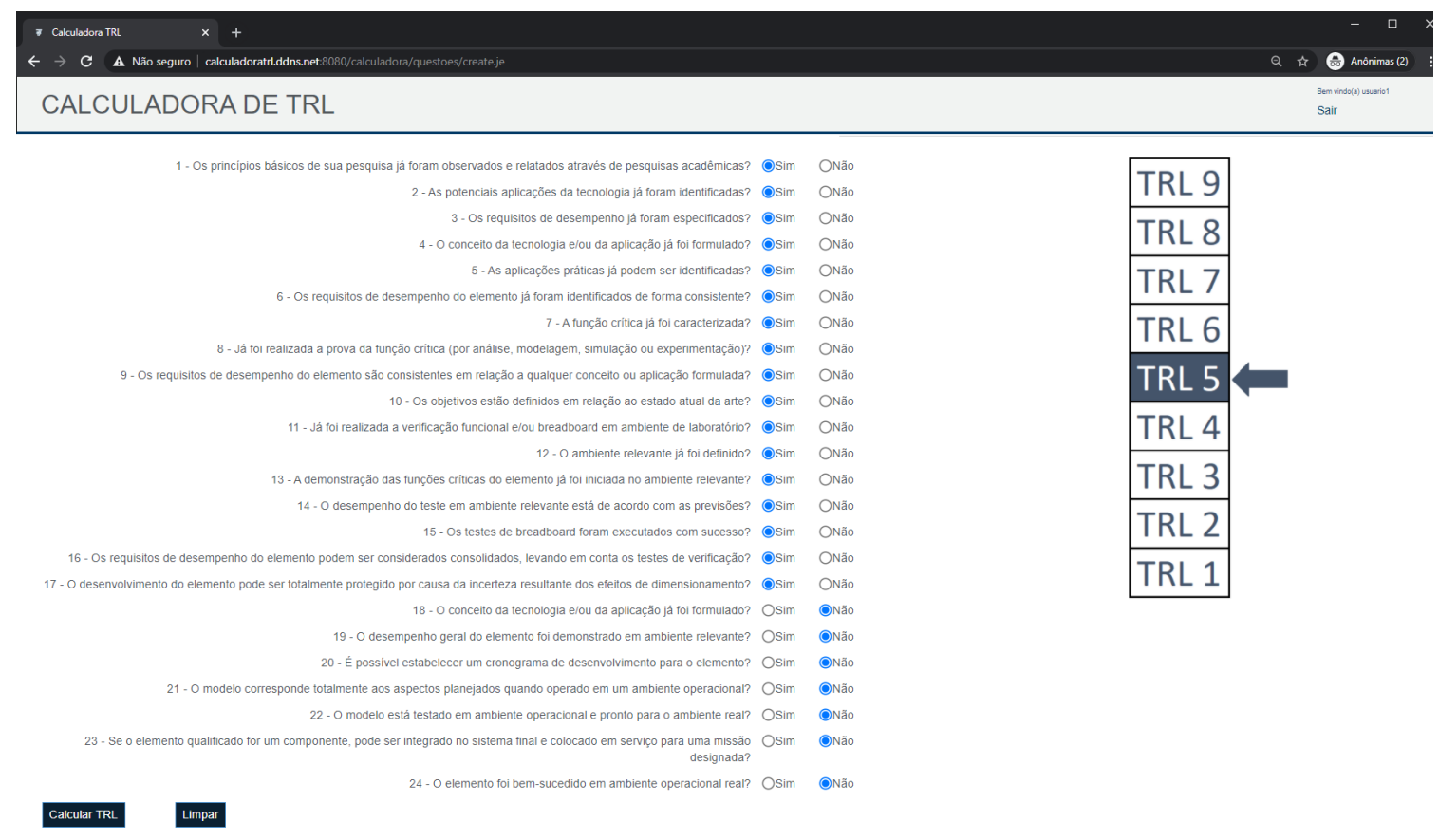

Fonte: Elaborada pelos autores deste artigo (2020) 
A Figura 3 apresenta a mensagem, em vermelho, que o usuário recebe em complemento ao resultado na escala, a qual informa as inconsistências nos níveis com respostas negativas.

Figura 3 - Simulação de resultado exato na escala

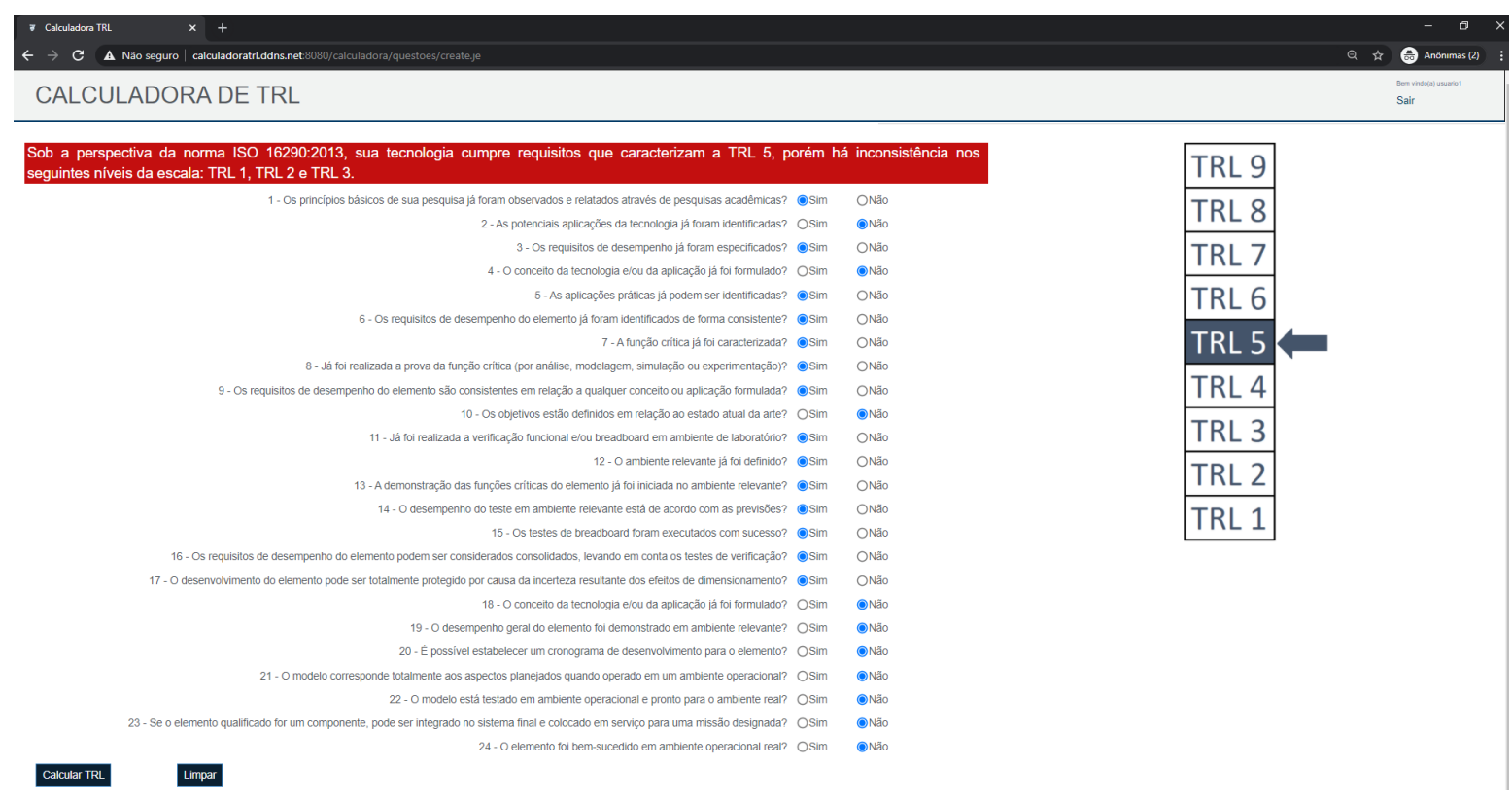

Fonte: Elaborada pelos autores deste artigo (2020)

Para analisar e classificar na TRL os pedidos de depósito de patentes da Universidade Federal de Santa Catarina, é importante contextualizar e apresentar a instituição. Trata-se de universidade pública e gratuita, sediada em Florianópolis, Santa Catarina, com outros quatro campi nas cidades de Araranguá, Blumenau, Curitibanos e Joinville, que desenvolve suas atividades baseadas nas dimensões do ensino, pesquisa e extensão.

Fundada em 1960, constitui-se atualmente de uma comunidade entre alunos de ensino básico, graduação e pós-graduação e servidores que amontam cerca de 70 mil pessoas (UFSC, 2020). De acordo com o RUF (2019), a UFSC figura o sétimo lugar no ranking geral, ocupando o $25^{\circ}$ lugar quanto à competitividade e $16^{\circ}$ em inovação.

A gestão da propriedade intelectual na universidade teve início com a criação do Núcleo de Inovação Tecnológica (NIT) em 1981. Em 2007, foi criado o Comitê de Inovação, com a função de acompanhar as ações de inovação e de pesquisa científica e tecnológica para a proposição de políticas de projetos, de propriedade intelectual, de segredo, de transferência de tecnologia e de incentivo à inovação. Em 2016, o núcleo incorporou novas atribuições, tornando-se uma agência de inovação. Entre essas novas atribuições, destaca-se a identificação no ambiente produtivo de oportunidades para realização de projetos de inovação para execução em conjunto com a UFSC e o estímulo à cultura do empreendedorismo na universidade. No mesmo ano, a agência modificou-se para Secretaria de Inovação, cuja missão atual é "[...] promover a inovação e o empreendedorismo, por meio de parcerias e interações com diferentes atores, criando condições para que o saber filosófico, científico, artístico e tecnológico produzido na Universidade, possa ser revertido em prol da sociedade" (SINOVA, 2021). 
As respostas ao formulário contendo o protocolo de classificação foram trabalhadas pelo software TRLCalc, produto deste estudo, para o enquadramento dos pedidos de depósito de patentes da Universidade Federal de Santa Catarina na escala TRL, o que revelou uma predominância de tecnologias prontas para exploração econômica.

Gráfico 1 - Classificação dos pedidos de depósito de patente da Universidade Federal de Santa Catarina

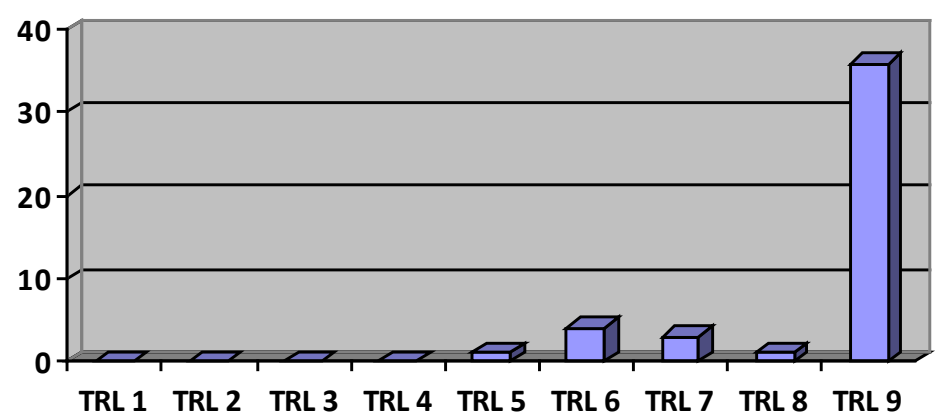

Fonte: Dados coletados pelos autores deste artigo e trabalhados no software TRLCalc (2020)

A partir do relatório enviado pela Secretaria de Inovação da universidade, departamento responsável pela gestão de sua propriedade intelectual, dos 120 pedidos de registro de patentes, foram obtidas 45 respostas, já descontadas as situações em que mais de um pesquisador respondeu sobre um mesmo pedido.

Das 45 patentes analisadas, 36 cumprem os requisitos para estarem classificadas no nono nível da escala, entre as quais, 16 apresentam resultado exato e as demais 24 apresentam inconsistências a partir do quinto nível da escala.

As nove patentes que não estão no último nível da escala distribuem-se a partir do quinto nível e apresentam, em sua maioria, inconsistências nos níveis anteriores aos de sua classificação final. Não houve resultados cujos atributos caracterizassem tecnologias a serem enquadradas do primeiro ao quarto níveis.

A universidade não apresenta em suas publicações institucionais um mapeamento de suas patentes já absorvidas pelo mercado, assim como não apresenta uma vitrine tecnológica que divulgue à comunidade as tecnologias aptas para a exploração econômica. Não é objetivo desta pesquisa compreender e especificar as razões da predominância de tecnologias classificadas no nono nível da escala.

Entretanto, é oportuno pontuar que o cenário das universidades brasileiras é impactado pela pontuação que a Capes ${ }^{10}$ confere aos pesquisadores quanto ao registro de propriedade intelectual, que, apesar de incrementar o currículo, não necessariamente manifesta de fato uma inovação, pois, muitas vezes, a invenção protegida não é transferida ao mercado. 
Tabela 1 - Distribuição da quantidade de patentes nos níveis da TRL por área do conhecimento Engenharia Elétrica (3), Engenharia Mecânica (17), Farmacologia (2) e Física (1)

TRL 8

Bioquímica (1)

TRL 7

Bioquímica (1), Engenharia Elétrica (1) e Engenharia Mecânica (1)

TRL 6

Engenharia Mecânica (1)

TRL 5

Engenharia Elétrica (1)

TRL 4

Não houve resultados

TRL 3

Não houve resultados

TRL 2

Não houve resultados

TRL 1

Não houve resultados

Fonte: Elaborada pelos autores deste artigo (2020)

A Tabela 1 apresenta a distribuição das patentes por área do conhecimento. É possível extrair dos dados uma predominância de patentes produzidas dentro do conhecimento da Engenharia Mecânica no nono nível da escala, ou seja, tecnologias já com seu sistema real comprovado por meio de operações de missão bem-sucedidas.

\section{Considerações Finais}

A classificação dos pedidos de depósito de patentes da UFSC na escala TRL, baseado na Norma ISO 16290:2013, a partir da solução tecnológica apresentada, revelou uma predominância de patentes localizadas no nono nível da escala, com inconsistências a partir do quinto nível. As informações sobre essas tecnologias, entretanto, encontram-se difusas, assim como ocorre com as demais universidades federais.

Os temas envolvidos na discussão da problemática deste estudo estão em plena expansão em termos de publicações recentes e a UFSC, atualmente, trabalha em uma proposta de sistematização de suas potenciais inovações. Essa sistematização possibilitará maior clareza e exatidão nas informações envolvidas no processo de transferência de tecnologia e aperfeiçoará a interação de arranjos complexos que envolvem as universidades, os governos e as empresas.

A aproximação entre universidade e setor produtivo requer o engajamento dos atores envolvidos no Sistema Nacional de Inovação. Por parte do governo, em suas diferentes esferas, o entendimento da inovação como grande motor do desenvolvimento nacional se mostra determinante. Por parte da universidade, há de se ter envolvimento para além da sua missão de produzir, com atuação na sistematização e na socialização do saber, aproximando áreas afins, simplificando procedimentos e melhorando a comunicação com a sociedade. Às empresas cabe entender a universidade, principalmente pública, como celeiro de oportunidades alinhadas às demandas locais e globais. 
O protocolo de classificação de patentes apresentado no presente estudo se revelou uma ferramenta acessível e de fácil manuseio pelos inventores, pois houve boa compreensão dos conceitos e das terminologias utilizadas e baixa ocorrência de solicitação de esclarecimento dos elementos utilizados. O software TRLCalc, ao relacionar a padronização e a segurança de uma norma ISO à possibilidade de se conhecer o nível de prontidão tecnológica de uma patente com potencial de mercado que a universidade produz, contribui para que as barreiras de comunicação entre os atores dos sistemas de inovação diminuam e para que se eleve a clareza $e$ exatidão de informações como fator relevante para comercialização.

\section{Perspectivas Futuras}

O software TRLCalc criado nesta pesquisa cumpre ao que se propõe, uma vez que se demonstrou uma ferramenta simples, confiável, de fácil utilização e que pode ser formatado para disponibilização em formato aplicativo mobile. Aprofundamentos complementares refinarão progressivamente os resultados.

Para trabalhos semelhantes que se dediquem a avaliar os estágios de desenvolvimento das tecnologias produzidas por centros de pesquisa, será útil o aprofundamento na influência do perfil do pesquisador nas respostas. Os dados analisados pelo presente estudo apontaram algumas diferenças de análise de pesquisadores diferentes para o mesmo projeto, o que pode revelar diferentes níveis de envolvimento e de compreensão do mesmo assunto.

Também é necessário que se apresente um mapeamento das inovações reais, ou seja, quais tecnologias obtiveram espaço no mercado, acompanhando seus ciclos de vida $e$ impactos nas organizações e sociedades em que se desenvolveram.

Estudos complementares poderão avaliar de que forma os diferentes arranjos institucionais são determinantes para que áreas específicas do conhecimento apresentem quantidades elevadas de registro de patentes em relação aos demais.

Análises futuras que repliquem a execução deste trabalho em outras universidades brasileiras poderão avaliar qual a influência da natureza da universidade ou instituto de pesquisa no desenvolvimento e produção de ativos tecnológicos com alta adesão ao mercado.

Será importante também verificar se a produção tecnológica de uma instituição de pesquisa está alinhada à necessidade regional em que se encontra e destacar quais fatores constroem a predominância de tecnologias mais maduras.

\section{Referências}

AGRAWAL, A. Engaging the inventor: exploring licencing strategies for university inventions and the role of latent knowledge. Strategic Management Journal, [s.l.], v. 27, n. 1, p. 63-79, 2006. DOI: 10.1002/smj.508.

AZEVEDO, P. A interação UFSC e Petrobrás para o desenvolvimento inovativo sob a óptica institucionalista-evolucionária. 2016. 508p. Tese (Doutorado) - Universidade Federal de Santa Catarina, Centro Socioeconômico, Programa de Pós-Graduação em Administração, Florianópolis, 2016. Disponível em: http://www.bu.ufsc.br/teses/PCAD0974-T.pdf. Acesso em: 17 set. 2020. 
BEN-AMI, P. Os riscos e as possibilidades de negócios. Pesquisa Fapesp, São Paulo, n. 50, p. 5-7, jan.-fev. 2000. (Encarte Especial Patentes).

CARAYANNIS, E. G.; CAMPBELL, D. F. J. 'Mode 3' and 'Quadruple Helix': toward a 21st century fractal innovation ecosystem. International Journal of Technology Management, [s.l.], v. 46, n. 3-4, p. 201-234, 2009.

CARAYANNIS, E. G.; CAMPBELL, D. F. J. Triple Helix: Quadruple Helix and Quintuple Helix and How Do Knowledge, Innovation and the Environment Relate To Each Other. [S.l.: s.n.], 2010.

CARAYANNIS, E. G.; BARTH, T. D.; CAMPBELL, D. FJ. The Quintuple Helix innovation model: global warming as a challenge and driver for innovation. Journal of Innovation and Entrepreneurship, [s.l.], v. 1, n. 1, p. 2, 2012.

CONSELHO NACIONAL DE DESENVOLVIMENTO CIENTÍFICO E TECNOLÓGICO (CNPq). Disponível em: http://cnpq.br/bioprospeccao-dt. Acesso em: 4 abr. 2018.

ETZKOWITZ, H.; LEYDESDORFF, L. A triple helix of university-industry-government relations. Londres: Continuum, 1997.

EUROPEAN COMISSION. Horizon 2020 - Work Programme 2014-2015. Disponível em: https:// ec.europa.eu/research/participants/data/ref/h2020/wp/2014_2015/annexes/h2020-wp1415-annexga_en.pdf. Acesso em: 2 jan. 2020.

ISO/TC 20/SC 14/FDIS 16290:2013. Space systems definition of the Technology Readiness Levels (TRLs) and their criteria of assessment. 2013.

LAKATOS, E. M.; MARCONI, M. A. Fundamentos da metodologia científica. São Paulo: Atlas, 2006.

LAPLANE, M. Inovações e dinâmica capitalista. In: CARNEIRO, R. Os Clássicos da Economia. São Paulo: Ática, 2004. v. 2. p. 59-67.

LOZANO, R. et al. Moving to a quintuple helix approach in SPP: Collaboration and LCC for lighting procurements. In: ANDHOV, M.; CARANTA, R.; WIESBROCK, A. (ed.) Cost and EU Public Procurement Law: Life-Cycle Costing for Sustainability, London: Routledge. 2019. p. 81-99.

MANKINS, J. C. Technology Readiness Levels: A White Paper, 1995. Disponível em: http://www. hq.nasa.gov/office/codeq/trl/trl.pdf. Acesso em: 11 fev. 2019.

OLECHOWSKI, A.; EPPINGER, S. D.; JOGLEKAR, N. Technology readiness levels at 40: A study of state-of-the-art use, challenges, and opportunities. In: PORTLAND INTERNATIONAL CONFERENCE ON MANAGEMENT OF ENGINEERING AND TECHNOLOGY. 2015. Anais [...]. Portland, 2015.

PLONSKI, G. A. Prefacio a la Cooperación Empresa-universidad. [S.l.: s.n.], 1992.

POSSAS, M. L. Em direção a um paradigma microdinâmico: a abordagem neoschumpeteriana. In: AMADEO, E. J. (org). Ensaios sobre economia a moderna: teoria e história do pensamento econômico. São Paulo: Marco Zero, 1989. p. 157-177.

PÓVOA, L. M. C.; RAPINI, M. S. Technology transfer from universities and public research institutes to firms in Brazil: what is transferred and how the transfer is carried out. Science and Public Policy, [s.l.], v. 37, n. 2, p. 147-159, 2015. DOI: 10.3152/030234210X496619. 
RUF - RANKING UNIVERSITÁRIO FOLHA. [2019]. Disponível em: https://ruf.folha.uol.com. br/2019/ranking-de-universidades/principal/. Acesso em: 5 out. 2020.

RIBEIRO, M. E. et al. TRLCalc: Calculadora de TRL. Versão 1.0. 21 out. 2020. Disponível em: http://calculadoratrl.ddns.net:8080/calculadora/login.je. Acesso em: 15 out. 2020.

SABATO, J.; BOTANA, N. La Ciencia y la Tecnología en el Desarrollo Futuro de America Latina. Revista de la Integración InTAL, [s.l.], v. 1, n. 3, 1968.

SADIN, S. The NASA Technology Push Towards Future Space Mission Systems. Acta Astronaut., [s.l.], v. 20, p. 73-77, 1989.

SECRETARIA DE INOVAÇÃO DA UFSC (SINOVA). SINOVA/UFSC. Disponível em: http://sinova. ufsc.br. Acesso em: 21 out 2020.

THURSBY, J.; FULLER, A.; THURSBY, M. US faculty patenting: inside and outside the university. Research Policy, [s.l.], v. 38, n. 1, p. 14-25, 2009. DOI: 10.1016/j.respol.2008.09.004.

UFSC - UNIVERSIDADE FEDERAL DE SANTA CATARINA. [2020]. Disponível em: http://www.ufsc.br. Acesso em: 16 out. 2020.

VERGARA, S. C. Projetos e relatórios de pesquisa em administração. São Paulo: Atlas, 2007.

\section{Sobre os Autores}

\section{Mariana Eleutério Ribeiro}

E-mail: mari.ribeiro@gmail.com ORCID: http://orcid.org/0000-0002-4120-8794

Mestre em Propriedade Intelectual e Transferência de Tecnologia para Inovação.

Endereço profissional: Seção de Controle e Acompanhamento Contratual - Tribunal de Justiça de Santa Catarina. Rua Álvaro Millen da Silveira, n. 208, Centro, Florianópolis, SC. CEP: 88020-901.

\section{Irineu Afonso Frey}

E-mail: irineu.inova@gmail.com

ORCID: http://orcid.org/0000-0002-7731-3406

Doutorado em Engenharia de Produção/UFSC

Endereço profissional: Universidade Federal de Santa Catarina, Departamento de Ciências Contábeis, Centro Socioeconômico, Campus Universitário, Trindade, Florianópolis, SC. CEP: 88040-970.

\section{Paola Azevedo}

E-mail: pazevedo4@gmail.com

ORCID: http://orcid.org/0000-0003-0312-8854

Doutorado em Administração pela UFSC.

Endereço profissional: Secretaria de Inovação (SINOVA), Avenida Desembargador Vitor Lima, n. 222, Loja 3 , Reitoria Prédio 2, Trindade, Florianópolis, SC. CEP: 88040-400. 